\title{
Effect of the Sedative Combination of Ketamine, Morphine and Midazolam on the Quality-of-Life in the Final Days of Terminally III Cancer Patients
}

\author{
Noam Asna ${ }^{1}$, Noa Shemesh ${ }^{2}$, Liat Arbel ${ }^{2}$, Rami Ben Yosef ${ }^{3}$, Ron Batash ${ }^{4}{ }^{*}$ and Moshe Schaffer ${ }^{1}$ \\ ${ }^{1}$ Department of Oncology, Barzilai Medical center, Ashkelon and Ben Gurion University of the Negev, Faculty of Health Sciences, Beer Sheva, Israel \\ ${ }^{2}$ Department of Oncology, Tel Aviv Sourasky Medical Center, Tel Aviv, Israel \\ ${ }^{3}$ Department of Oncology, Rambam Health Care Campus, Haifa, Israel \\ ${ }^{4}$ Department of Orthopedics, Barzilai Medical center, Ashkelon and Ben Gurion University of the Negev, Faculty of Health Sciences, Beer Sheva, \\ Israel
}

*Corresponding author: Ron Batash, Department of Orthopedics, Barzilai Medical center, Ashkelon, and Ben Gurion University of the Negev, Faculty of Health Sciences, Beer Sheva, Israel, Tel: 97286746037; Fax: 97286746141; E-mail: Ron.batash@gmail.com

Received date: April 25, 2017; Accepted date: April 27, 2017; Published date: April 29, 2017

Citation: Asna N, Shemesh N, Arbel L, et al. Effect of the Sedative Combination of Ketamine, Morphine and Midazolam on the Quality-of-Life in the Final Days of Terminally III Cancer Patients. Arch Med. 2017, 9:2

Copyright: (c) 2017 Asna N, et al. This is an open-access article distributed under the terms of the Creative Commons Attribution License, which permits unrestricted use, distribution, and reproduction in any medium, provided the original author and source are credited.

\section{Abstract}

Background: Sedation in terminally-ill cancer patients is typically used to alleviate symptoms of physical distress during the last week of life, in order to allow patients to achieve serenity. These include pain, as well as respiratory, neuropsychiatric, and gastrointestinal symptoms.

The purpose of this study is to investigate the effect of the sedative protocol containing ketamine, midazolam, and morphine (KMM) on symptoms of terminally-ill cancer patients at the end-of-life, as well as on consciousness level, and overall survival.

Methods and Findings: This retrospective study included terminally-ill cancer patients ( $>18$ years) who completed cancer-specific treatment and received KMM upon hospitalization. Medical records were reviewed. The symptoms were classified according to their severity before and during treatment. Control of debilitating symptoms and level of consciousness during the hospitalization was clinically evaluated.

Results: The study included 30 patients (10 males; mean age, 55 years). Range doses were: $0.1-1 \mathrm{mg} / \mathrm{kg}, 0.05-0.5$ $\mathrm{mg} / \mathrm{kg}$, and 0.5-5 mg/kg daily, for ketamine, midazolam, and morphine, respectively. The mean survival was 4.6 days (therefore, analyses focused on the first 3 days). A statistically significant decrease in pain was achieved on the first day of treatment $(P<0.0001)$. Further improvement was sustained through the following 2 days $(P=0.022)$. An improvement in respiratory, gastrointestinal, and neuropsychiatric symptoms was reported $(P=0.033)$. A significant decline in consciousness level was observed with time. The initial pain level (before administrating KMM) was negatively associated with survival.
Conclusions: The KMM protocol is an effective method in controlling pain and providing relief from respiratory, neuropsychiatric, and gastrointestinal symptoms. No KMMassociated complications were identified. Further research is warranted.

Keywords: End-of-life; Ketamine; Metastatic cancer; Midazolam; Morphine; Pain; Palliative care

\section{Introduction}

Sedation in terminally-ill patients is typically used to alleviate symptoms of physical distress during the last week of life in order to allow patients to achieve serenity. These include significant pain or fatigue (reported in approximately $70 \%$ of patients), agitation, restlessness, delirium or acute respiratory symptoms $(60 \%)$, urinary retention or incontinence $(50 \%)$, dyspnea (20\%), nausea and vomiting (10\%), and other gastrointestinal (GI) symptoms [1,2]. The most commonly-used sedatives are benzodiazepines, either with or without opiates.

No consensus exists as to the classification of terminally-ill patients, and there are no clear guidelines for time limits with respect to sedative treatment in such patients [3]. Moreover, data on the effect of such treatment on the clinical implications of supportive care, on patient monitoring, or on other factors such as hydration and nutritional status are limited $[4,5]$. Furthermore, it is unclear at what stage of disease should this treatment be offered, and when to obtain informed consent from the patients or their families [6].

There is a broad agreement that sedatives must be used to treat the aforementioned symptoms, despite their association with reduced life expectancy. Notably, some studies have shown that administration of some traditional palliative drugs (e.g., opiates) did not have a significant impact on life expectancy [7-9]. One of the most common combinations of first-line 
sedation in oncology is morphine plus midazolam. This combination may be administered alone or as part of analgesicsedative combinations that include morphine. An additional combination includes ketamine plus midazolam, where midazolam prevents the psychotic effects of ketamine. Coadministration of ketamine and morphine was found to be more effective than morphine alone in reducing pain [10]. Adding midazolam to this combination prevented hallucinations in patients who were treated by either morphine alone or by morphine and ketamine, with no major complications [10]. Also, administrating ketamine and morphine to 8 terminally-ill patients with bone metastases and opiate tolerance was found to be effective [11]. Other studies demonstrated that adding midazolam to morphine helped in alleviating severe dyspnea perception [12]. Although opiates have anti-anxiety effects initially, midazolam may contribute to this effect as tolerance to opiates develops during treatment [13].

The objective of this retrospective study was to evaluate the effect of a regimen containing ketamine, morphine, and midazolam (KMM) on the quality-of-life of cancer patients during the terminal stage of their disease.

\section{Methods}

\section{Study design}

This retrospective study included 30 terminally-ill patients who were hospitalized at the Tel Aviv Sourasky Medical Center between January 2009 and December 2013 due to severe symptoms of cancer, and were treated with a KMM protocol.

Institutional Review Board (IRB) and Cancer Center Research Review committee approvals were obtained. The study was granted a waiver for obtaining informed consent.

\section{Eligibility}

The study included patients (males and females) $>18$ years of age with proven diagnosis of cancer who had reached the terminal stage of the disease (defined as $<8$ days of expected life expectancy upon admission). Additional eligibility criteria were: completion of all cancer-specific treatment, receiving supportive care only, and developing symptoms including uncontrolled pain under strong analgesics, acute respiratory difficulties without a decrease in consciousness, decrease in consciousness, delirium and psychomotor restlessness, or massive and uncontrollable hemorrhage, for which patients received KMM treatment.

\section{Treatments}

All patients included in the study received $\mathrm{KMM}$ regimen as follows: intravenous (IV) ketamine $0.1-1 \mathrm{mg} / \mathrm{kg} /$ day, IV morphine 0.5-5 mg/kg/day, and IV midazolam 0.05-0.5 mg/kg/day. In addition, all patients received a laxative in order to prevent constipation due to morphine treatment.

\section{Data collection}

Patient demographics (age, gender) and the number of hospitalization days until death were collected from the medical records. In addition, the following parameters were collected and evaluated by experienced oncology nurses: level of consciousness (1-4 scale: 1, conscious and cooperating; 2, alternating being asleep and awake and communicates often; 3 , mostly asleep and is difficult to communicate with; 4, asleep and is difficult to wake up or to communicate with); respiratory difficulty status (0-4 scale: 0 , none; 1 , minor; 2 , moderate; 3 , difficult; 4, hard to evaluate) including breathlessness/dyspnea, terminal respiratory congestion, pleural effusion, cough, and lung edema; GI symptoms (0-4 scale: 0, none; 1, minor; 2, moderate; 3, difficult; 4, hard to evaluate) including nausea, vomiting, dysphagia, loss of appetite, constipation/diarrhea, bleeding, and bowel occlusion; neuropsychiatric symptoms (0-4 scale: 0 , none; 1 , minor; 2 , moderate; 3 , difficult; 4 , hard to evaluate) including confusion, depression, anxiety, restlessness, delirium, insomnia/hypersomnia, and hallucinations. Pain characteristics recorded included level of pain using visual analog scale (VAS, 0-10 scale), type, and location. Additional parameters collected included nutrition (total parenteral nutrition [TPN]/food/liquids/IV fluids), mobility, peripheral edema or ascites, type of malignancy, and previous analgesics. Each patient was evaluated 3 times a day (once in each shift), and the average of the evaluations was used for the analysis.

\section{Statistical analysis}

Descriptive statistics was used to summarize the data. McNemar's test was used to analyze the effect of KMM on each group of symptoms, some of which were coded as binary variables (i.e., presence vs. absence of symptoms), and some by severity. The effect of KMM on pain was analyzed using analysis of variance (ANOVA) with repeated measurements. Univariate comparisons of potential prognostic factors for survival were calculated using the log-rank analysis and Kaplan-Meier curves. $P$ values were all 2-tailed; $P<0.05$ was considered statistically significant.

\section{Results}

The study included 30 patients (20 females [66\%], 10 [34\%] males). The mean ( \pm SD) age was $55.6 \pm 14.0$ years (range, $38-90)$. The mean ( \pm SD) survival of patients after hospitalization was $4.6 \pm 1.9$ days (range 2-8.7). All patients were previously exposed to analgesics.

The mean ( \pm SD) doses of medications given within hospitalization for ketamine, morphine, and midazolam, were $578.3 \pm 432.1 \mathrm{mg}$ (range, 5-1500), $490 \pm 532.6 \mathrm{mg}$ (range, $100-1,500)$, and $24.5 \pm 18.4 \mathrm{mg}$ (range, 5-60), respectively. These doses were administered at the discretion of the treating oncologist and according to the previous exposure of the patients to treatments and pain level of the patient. The average dose increased during treatment. Due to the short mean survival and consequently to the decrease in sample size with time, the statistical analysis was limited to the first 3 days of hospitalization. 


\section{Effect of the KMM combination on pain}

A mean $( \pm S D$ ) reduction in pain (as measured using 0-10 VAS) of $3.7 \pm 2.3$ points was reported after a day of KMM treatment. After 3 days of KMM treatment, pain significantly decreased by 5.4 points (from a mean of $7.2 \pm 1.4$ to $1.8 \pm 2.8$ points; $\mathrm{P}<0.0001$, Figure 1). Stratification of pain according to location or type was not possible as most patients suffered from somatic as well as neuropathic pain.

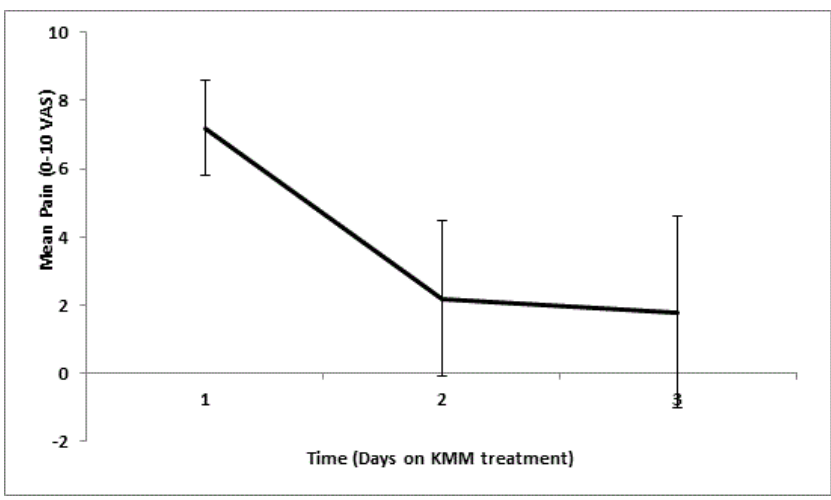

Figure $1 \mathrm{Effect}$ of $\mathrm{KMM}$ on pain $(\mathrm{N}=30)$. Error bars represent SD; 0-10 VAS was used for reporting pain.

\section{Effect of the KMM combination on consciousness level}

After a day of KMM treatment, a nonsignificant decrease \pm SD) in consciousness level was observed $(0.17 \pm 1.9$ points using a 1-4 consciousness scale). After 3 days of KMM treatment, a significant decrease in consciousness was observed (from $1.3 \pm$ 0.5 to $2.4 \pm 1.1$ points; $P<0.0001$; Figure 2 ).

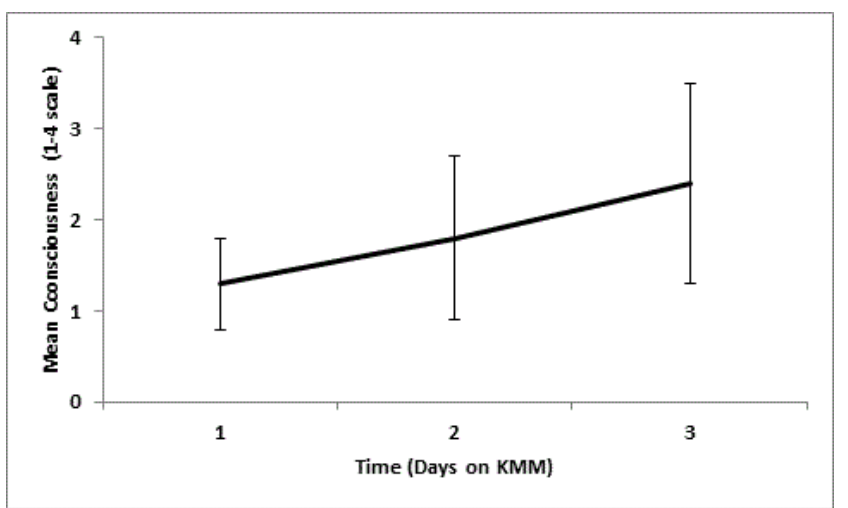

Figure 2 Effect of KMM on consciousness $(\mathrm{N}=30)$. Error bars represent SD; consciousness levels were reported using a 1-4 scale (higher values represent lower consciousness level).

\section{Respiratory symptoms}

Twenty patients $(66.0 \%)$ had respiratory symptoms on the first day of hospitalization. For 11 of them, (55.0\%), respiratory symptoms improved on the second hospitalization day $(\mathrm{P}=0.022)$.

Two out of 10 patients who were initially without respiratory symptoms on the first day, developed respiratory symptoms that were improved during KMM treatment.

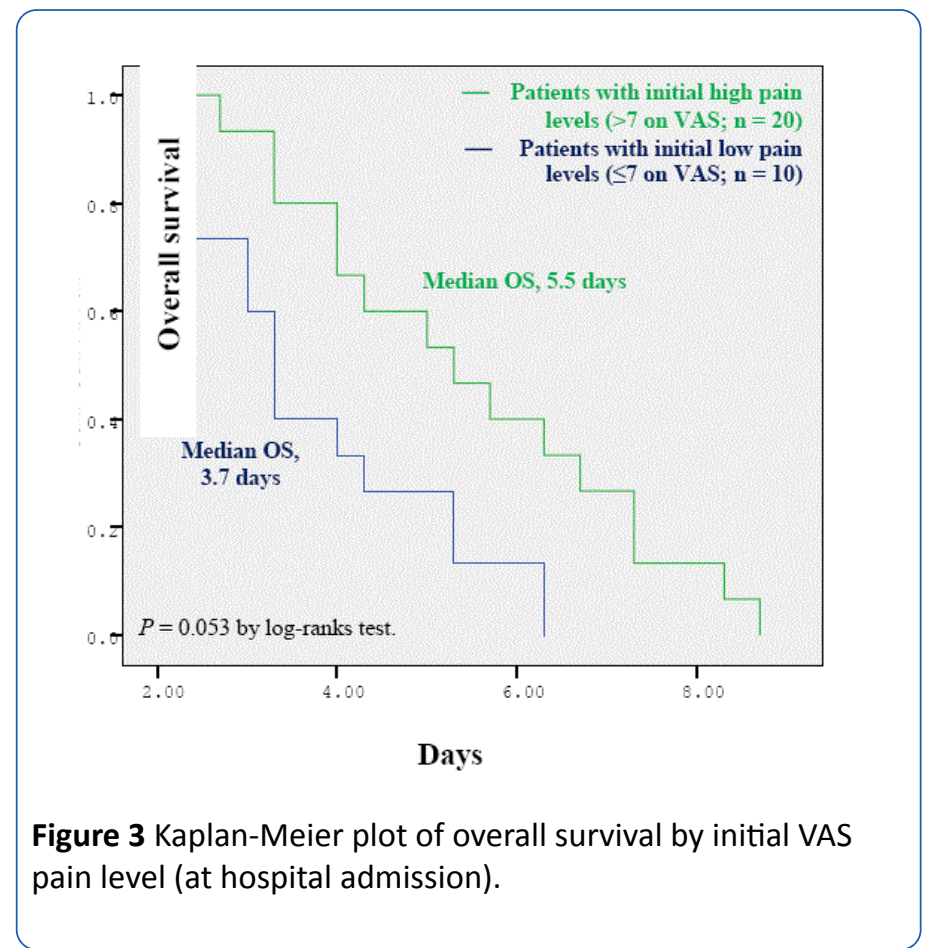

\section{Neuropsychiatric symptoms}

The vast majority of patients (29 of $30,96.7 \%$ ) experienced neuropsychiatric symptoms on their first hospitalization day. One patient was asymptomatic and remained so during the 3 evaluated days of hospitalization. A statistically significant improvement $(P=0.031)$ in neuropsychiatric symptoms was observed in 6 of $29(20.7 \%)$ patients between the first and second hospitalization day. Of the 23 patients who did not improved by the second day, 2 patients $(8.7 \%)$ experienced improvement in symptoms between the second and third day. None of the patients experienced worsening of symptoms. Between the first and third day, a nonsignificant statistically improvement was reported.

\section{Gastrointestinal symptoms}

Twenty-two patients had Gl symptoms on the first day of hospitalization. Of these, one (4.5\%) improved between the first and second day. Between the second and third hospitalization day, GI symptoms in 6 of 21 patients (28.6\%) improved $(P=0.031)$. Overall, during the first 3 days of hospitalization, 7 of the 22 patients (31.8\%) who suffered Gl symptoms on the first day, experienced improvement in their Gl symptoms.

Only 3 patients had constipation on the first hospitalization day, and none reported diarrhea. During the 3 days of the study, the constipation in these patients remained and no additional patients reported constipation or diarrhea. 


\section{Survival analysis}

Since pain was the most prevalent symptom, we analyzed overall survival by the level of initial (pre-KMM) VAS pain. This Kaplan-Meier survival analysis showed a trend towards longer survival in patients who had initial high level of pain ( $>7$ on VAS; $n=20)$ compared with those $(n=10)$ with initial lower pain levels ( $\leq 7$ on VAS) (5.5 vs. 3.7 days; $\mathrm{P}=0.053$ ) (Figure 3).

\section{Discussion}

This observational retrospective study examined the effect of KMM treatment on various symptoms in terminally-ill cancer patients. We found that the mean survival was 4.6 days and that the KMM protocol was effective in reducing pain, as well as in improving respiratory, neuropsychiatric, and Gl symptoms. We also observed a significant decline in consciousness level over the duration of the study. There was a trend $(P=0.053)$ suggesting a negative correlation between the initial pain level (before KMM administration) and survival.

Most of the studies that investigated opiate-ketamine combinations had small sample sizes and focused only on pain [10]. Our objective was to study the KMM protocol, where midazolam, which is generally given to treat adverse events, is administered from the first day of hospitalization. We studied the effect of KMM on pain as evaluated by VAS, which is considered a reliable pain assessment tool in oncology [14]. In addition, we examined the effect of $\mathrm{KMM}$ on a wide range of systems/symptoms in terminally-ill patients and on patients level of consciousness, which is an important issue at the end-oflife (as families often feel the need for more time with the dying patient). Lastly, we also examined the effect of KMM on survival, as the issue of quality-of-life versus life expectancy is of increasing relevance at the end-of-life $[15,16]$. Notably, our results should be interpreted cautiously since this is a retrospective study (a controlled trial in this setting is ethically complicated) [17]; for example, we found that consciousness levels declined steadily, however, this may be due to the natural course of the disease, not KMM.

KMM treatment was associated with improvement in many systems. The observed improvement in respiratory symptoms is remarkable considering the expected respiratory worsening in all patients and the known adverse effects of morphine as a respiratory suppressant. GI symptoms also improved significantly and no patient had diarrhea or constipation as a result of KMM treatment. Nonetheless, KMM did not improve constipation symptoms in the 3 patients who experienced them prior to KMM treatment.

The vast majority of patients experienced neuropsychiatric symptoms throughout the study. The frequency of these symptoms suggests that they may have triggered the hospitalization of our patients, as families are often unable to address such symptoms in home care. On the other hand, such symptoms may simply reflect the status of these terminally-ill patients. Overall, we observed improvement in neuropsychiatric symptoms in more than a quarter $(28 \%)$ of patients between the first and third hospitalization day. This improvement may also be attributed to an indirect effect of KMM, for example, improvement in respiratory symptoms (i.e., alleviating anoxia). In addition pain reduction improved delirium [18]. Notably, neuropsychiatric symptoms were not reported in patients whose symptoms improved suggesting that the KMM protocol did not cause any neuropsychiatric adverse events in this patient population.

The association between pain management and survival is a debated issue. Respiratory suppression due to opiate administration may occur within several days and good control of pain was found to be associated with shorter survival [19]. On the other hand, several studies have shown a positive correlation between pain relief and life expectancy [20]. In our study, the only significant association with survival was for VAS pain level upon hospitalization. Surprisingly, patients with higher pain levels survived longer than those with lower pain levels. It is unclear if this observation is associated with the KMM protocol, but it does support the rationale of using KMM in patients who suffer more pain. Further research is needed to evaluate pain as a prognosticator of life expectancy in terminally-ill patients (thus far, most research efforts to identify prognosticators in this population focused on objective plasma parameters such as leucocytes, lactate dehydrogenase, etc) [21].

The limitations of this study include possible selection bias (hospitalized patients may have other characteristics than hospice patients). Notably, we observed the effect of KMM in hospitalized patients who tend to have more complications than hospice patients; therefore, it could be that KMM may be even more effective in patients with fewer complications (i.e., hospice patients). On the other hand, the oncology department is more equipped than hospice to treat complications, and therefore, we monitored all the complications that may have resulted from KMM. We found no KMM-associated complications; however, the status of the patients may have obscured the adverse effect of the KMM protocol. Another limitation is that our assessment, in part, was subjective and challenging as communication was an issue with some patients, especially those who required higher doses medications. To address these limitations, measurements were averaged per day (one evaluation per shift), and only experienced nursing staff performed the evaluation. Another weakness stems from the small sample size that decreased further over time, thereby limiting our statistical analyses to the first 3 days of hospitalization. Thus, later effects of KMM may have been overlooked. Notably, as the level of consciousness decreased over time, so did the need for palliation (as well as the ability to evaluate various symptoms); therefore, the first 3 days are the most critical for palliative care. Lastly, this was a retrospective uncontrolled study, and therefore, the KMM protocol cannot be compared to either no treatment or to other treatments.

In conclusion, the KMM protocol was an effective paincontrolling tool and provided relief from respiratory, neuropsychiatric, and GI symptoms in cancer patients at the end-of-life. No KMM-associated complications were identified. Further research on the role of this protocol at the end-of-life is warranted. 


\section{References}

1. Coyle N, Adelhardt J, Foley KM, Portenoy RK (1990) Character of terminal illness in the advanced cancer patient: pain and other symptoms during the last four weeks of life. J Pain Symptom Manage 5: 83-93.

2. Fainsinger RL, Waller $A$, Bercovici $M$, Bengtson $K$, Landman $W$, et al. (2000) A multicentre international study of sedation for uncontrolled symptoms in terminally ill patients. Palliat Med 14: 257-265.

3. Hallenbeck JL (2000) Terminal sedation: Ethical implications in different situations. J Palliat Med 3: 313-320.

4. Viola RA, Wells GA, Peterson J (1997) The effects of fluid status and fluid therapy on the dying: a systematic review. J Palliat Care 13: 41-52.

5. De Graeff A, Dean M (2007) Palliative sedation therapy in the last weeks of life: a literature review and recommendations for standards. J Palliat Med 10: 67-85.

6. Cherny NI, Coyle N, Foley KM (1996) Guidelines in the care of the dying cancer patient. Hematol Oncol Clin North Am 10: 261-286.

7. Stone P, Phillips C, Spruyt O, Waight C (1997) A comparison of the use of sedatives in a hospital support team and in a hospice. Palliat Med 11: 140-144.

8. Cowan JD, Palmer TW (2002) Practical guide to palliative sedation. Curr Oncol Rep 4: 242-249.

9. Chiu TY, Hu WY, Lue BH, Cheng SY, Chen CY (2001) Sedation for refractory symptoms of terminal cancer patients in Taiwan. J Pain Symptom Manage 21: 467-472.

10. Bell RF, Eccleston C, Kalso E (2003) Ketamine as adjuvant to opioids for cancer pain. A qualitative systematic review. J Pain Symptom Manage 26: 867-875.
11. Chazan S, Ekstein MP, Marouani N, Weinbroum AA (2008) Ketamine for acute and subacute pain in opioid-tolerant patients. J Opioid Manag 4: 173-180.

12. Navigante AH, Cerchietti LC, Castro MA, Lutteral MA, Cabalar ME (2006) Midazolam as adjunct therapy to morphine in the alleviation of severe dyspnea perception in patients with advanced cancer. J Pain Symptom Manage 31: 38-47.

13. Thomas JR, von Gunten CF (2002) Clinical management of dyspnoea. Lancet Oncol 3: 223-228.

14. Jensen MP (2003) The validity and reliability of pain measures in adults with cancer. J Pain 4: 2-21.

15. Kuhl D, Stanbrook MB, Hebert PC (2010) What people want at the end of life. CMAJ 182: 1707.

16. Ferris FD (2004) Last hours of living. Clin Geriatr Med 20: 641-667.

17. Ellis PM (2000) Attitudes towards and participation in randomised clinical trials in oncology: a review of the literature. Ann Oncol 11: 939-945.

18. Maldonado JR (2008) Delirium in the acute care setting: characteristics, diagnosis and treatment. Crit Care Clin 24: 657-672.

19. Desandre PL, Quest TE (2010) Management of cancer-related pain. Hematol Oncol Clin North Am 24: 643-658.

20. Staats PS, Hekmat H, Sauter P, Lillemoe K (2001) The effects of alcohol celiac plexus block, pain, and mood on longevity in patients with unresectable pancreatic cancer: A double-blind, randomized, placebo-controlled study. Pain Med 2: 28-34.

21. Hyodo I, Morita T, Adachi I, Shima Y, Yoshizawa A, et al. (2010) Development of a predicting tool for survival of terminally ill cancer patients. Jpn J Clin Oncol 40: 442-448. 